\title{
A GENERAL CHARACTERIZATION OF ONE FACTOR AFFINE TERM STRUCTURE MODELS
}

\author{
DAMIR FILIPOVIĆ
}

\begin{abstract}
We give a complete characterization of affine term structure models based on a general nonnegative Markov short rate process. This applies to the classical CIR model but includes as well short rate processes with jumps. We provide a link to the theory of branching processes and show how CBIprocesses naturally enter the field of term structure modelling. Using Markov semigroup theory we exploit the full structure behind an affine term structure model and provide a deeper understanding of some well-known properties of the CIR model. Based on these fundamental results we construct a new short rate model with jumps, which extends the CIR model and still gives closed form expressions for bond options.
\end{abstract}

\section{INTRODUCTION}

It is well known that the Cox-Ingersoll-Ross (CIR) short rate model possesses an affine term structure (ATS)

$$
P(t, T)=e^{-A(T-t)-B(T-t) r_{t}},
$$

see [5]. Conversely, it has been shown in [3] and [7] that any diffusion (and hence continuous) nonnegative short rate process $r=\left(r_{t}\right)_{t \geq 0}$ providing an ATS is necessarily of the CIR type. All these proofs are based on Itô calculus, not taking into account the rich and strong structure behind an ATS.

However, interest rates do not evolve continuously over time. Any model yielding solely continuous trajectories $t \mapsto r_{t}(\omega)$ should be considered only as a simplified description of the real behavior of the interest rates. Coherent models include jumps.

In this paper we characterize the class of all (including non-continuous) nonnegative Markov short rate processes $r$ which yield an ATS. Indeed, we start with any time homogeneous progressively measurable Markov process $r$ on $\mathbb{R}_{+}$which satisfies the natural condition that $\int_{0}^{t} r_{s} d s$ is finite a.s. for all $t$. Assuming an ATS, we then show - using Markov semigroup theory - that $r$ is necessarily a Feller process with generator

$$
\begin{aligned}
\mathcal{A} f(x)= & \alpha x f^{\prime \prime}(x)+\left(b^{\prime}+\beta x\right) f^{\prime}(x) \\
& +\int_{\mathbb{R}_{+}^{\circ}}\left(f(x+y)-f(x)-f^{\prime}(x)(1 \wedge y)\right)(m(d y)+x \mu(d y)),
\end{aligned}
$$

Date: September 9, 1999 (first draft); January 31, 2001 (this draft).

1991 Mathematics Subject Classification. Primary: 90A09; Secondary: 60J85.

Key words and phrases. affine term structure models, CBI-processes, infinitely decomposable processes, non-continuous Markovian short rates. 
where $\alpha, b^{\prime}, \beta, m(d y)$ and $\mu(d y)$ are parameters to be specified below (see Theorem 5.3). On the first line stands the continuous CIR part. On the second line there enters the jump part. The Levy measure, as well as the diffusion and the drift part, are seen to depend linearly on the state variable $x$. Such processes are known as (stochastically continuous) conservative CBI-processes (continuous state branching processes with immigration) and have been well studied, among others, by Kawazu and Watanabe in [16].

Conversely, any operator $\mathcal{A}$ of the above form generates in a unique way a conservative Feller process $r$ on $\mathbb{R}_{+}$providing an ATS model. Thus we establish a one to one correspondence between the class of conservative CBI-processes (describing branching phenomena) and Markov short rate processes implying an ATS, respectively.

For valuing interest rate sensitive instruments indispensable is the pricing semigroup

$$
Q_{t} f(x)=\int_{\mathbb{R}_{+}} f(y) q_{t}(x, d y):=\mathbb{E}\left[e^{-\int_{0}^{t} r_{s} d s} f\left(r_{t}\right) \mid r_{0}=x\right] .
$$

We identify $Q_{t}$ in terms of the Laplace transform of its transition function $q_{t}(x, d y)$. Thus by Laplace inversion we get (in principle) closed form expressions for all European claims $f\left(r_{t}\right)$. This explains a known fact of the CIR model.

We also restate a result from Shiga and Watanabe [21] that any conservative CBI-process is infinitely decomposable and vice versa. This considerably extends and clarifies another well known fact behind the CIR model, namely the additivity property of the squared Bessel processes

$$
\mathbb{Q}_{x}^{\delta} * \mathbb{Q}_{x^{\prime}}^{\delta^{\prime}}=\mathbb{Q}_{x+x^{\prime}}^{\delta+\delta^{\prime}}
$$

see [18, Chapt. XI.1]. As a byproduct we observe that any conservative CBI - and hence any infinitely decomposable - process is a semimartingale.

Based on these fundamental results we fully work out a non-continuous short rate model which extends the classical CIR model and still gives closed form expressions for European bond option prices. This model has already been discussed and applied in [6].

In the multifactor case, however, ATS does no longer imply affinity of the underlying Markovian factors in general. As a counter example we present a two factor ATS model driven by a two dimensional non-affine diffusion process.

A different approach towards the characterization of ATS models can be found in Duffie, Pan and Singleton [8], including the discussion on multifactor models. They also provide examples which are similar to the present one. See also [2] for sufficient conditions for an ATS in a jump-diffusion short rate model.

The remainder of the paper is organized as follows. In Section 2 we restate some of the basic facts about Markov processes and give the general setup for a Markov short rate model. In Section 3 we restrict our considerations to those models which provide an ATS. We discuss first structural consequences and derive a regularity result: the above pricing semigroup $\left(Q_{t}\right)$ is Feller. Section 4 contains the definition of a CBI-process and provides a summary of the results in [16] and [21]. In Section 5 we state our main result: the complete characterization of the class of Markov short rate processes which provide an ATS. In Section 6 we derive the pricing formula for a European bond call option within our framework. Section 7 presents a worked out model including jumping short rates. We give the explicit expressions for the bond 
option prices. In Section 8 we discuss shortly the multifactor case and provide a counter-example. We then conclude by Section 9. The appendix contains the basic lemma for the characterization of CBI-processes.

Terminology and Notation. Basically, we follow the notation of [18]. We write $\mathbb{R}_{+}=[0, \infty), \mathbb{R}_{+}^{\circ}=(0, \infty)$, and $\overline{\mathbb{R}}_{+}=[0, \infty]$ for the one point compactification of $\mathbb{R}_{+}$.

Denote by $C\left(\mathbb{R}_{+}\right)$and $C_{b}\left(\mathbb{R}_{+}\right)$the spaces of continuous and uniformly bounded continuous functions on $\mathbb{R}_{+}$, respectively. We write $C_{0}\left(\mathbb{R}_{+}\right)$and $C_{c}\left(\mathbb{R}_{+}\right)$for the spaces of elements in $C\left(\mathbb{R}_{+}\right)$vanishing at infinity and having compact support, respectively. The space of $k$ times continuously differentiable functions on $\mathbb{R}_{+}$is denoted by $C^{k}\left(\mathbb{R}_{+}\right)$. Analogously, we write $C_{b}^{k}\left(\mathbb{R}_{+}\right), C_{0}^{k}\left(\mathbb{R}_{+}\right)$and $C_{c}^{k}\left(\mathbb{R}_{+}\right)$.

Let $\mathcal{R}_{+}$denote the Borel $\sigma$-algebra on $\mathbb{R}_{+}$and $B\left(\mathbb{R}_{+}\right)$the space of bounded $\mathcal{R}_{+}$-measurable functions.

Equipped with the norm $\|f\|:=\sup _{x \in \mathbb{R}_{+}}|f(x)|$ the sets $C_{b}\left(\mathbb{R}_{+}\right), C_{0}\left(\mathbb{R}_{+}\right)$and $B\left(\mathbb{R}_{+}\right)$are Banach spaces.

\section{Markovian Short Rates}

In this section we provide the setup for a Markov short rate model. For the following definitions we refer to [18, Chapt. III.1] and [9, Chapt. I-II].

Let $r=\left(r_{t}\right)_{t \geq 0}$ be an $\mathbb{R}_{+}$-valued homogeneous Markov process, given by a transition function $p_{t}(x, d y)$ on $\left(\mathbb{R}_{+}, \mathcal{R}_{+}\right)$. We will assume that $r_{t}(\omega)=\omega(t)$ is realized as coordinate process on the canonical space $\Omega=\mathbb{R}_{+}^{[0, \infty)}$ with its natural filtration $\left(\mathcal{F}_{t}\right)_{t \geq 0}$ and $\mathcal{F}=\vee_{t \in \mathbb{R}_{+}} \mathcal{F}_{t}$. Thus, we are given a family of probability measures $\left(\mathbb{P}_{x}\right)_{x \in E}$ on $(\Omega, \mathcal{F})$ with $\mathbb{P}_{x}\left[r_{0}=x\right]=1$, such that $r$ is Markov under each $\mathbb{P}_{x}$.

The corresponding semigroup $\left(P_{t}\right)$ on $B\left(\mathbb{R}_{+}\right)$, see $[9$, Chapt. II.1], is given by

$$
P_{t} f(x)=\int_{\mathbb{R}_{+}} f(y) p_{t}(x, d y)=\mathbb{E}_{x}\left[f\left(r_{t}\right)\right], \quad x \in \mathbb{R}_{+}, \quad f \in B\left(\mathbb{R}_{+}\right) .
$$

Here $\mathbb{E}_{x}$ denotes expectation with respect to $\mathbb{P}_{x}$. We define the shift operators $\theta_{t}: \Omega \rightarrow \Omega$ by $\left(\theta_{t} \omega\right)(s)=\omega(t+s)$. Then the Markov property reads

$$
\mathbb{E}_{x}\left[Z \circ \theta_{t} \mid \mathcal{F}_{t}\right]=\mathbb{E}_{r_{t}}[Z], \quad \mathbb{P}_{x} \text {-a.s. } \quad \forall x \in \mathbb{R}_{+},
$$

for any nonnegative or bounded $\mathcal{F}$-measurable random variable $Z$.

Notice that this is a fairly general setup for a time homogeneous Markov process in $\mathbb{R}_{+}$. However, we want the savings account $e^{\int_{0}^{t} r_{s} d s}$ to be a well-defined $\mathbb{R}_{+-}$ valued adapted process, for each $\mathbb{P}_{x}$. This is equivalent to the following standing assumption:

(A1): The process $r$ is progressively measurable and

$$
\int_{0}^{t} r_{s} d s<\infty, \quad \mathbb{P}_{x} \text {-a.s. } \quad \forall t, x \in \mathbb{R}_{+} .
$$

This implies in particular that $r$ is conservative, i.e. $p_{t}\left(x, \mathbb{R}_{+}\right)=1$ for all $t$ and $x$.

It is clear that $\left(P_{t}\right)$ is a positive contraction semigroup on $B\left(\mathbb{R}_{+}\right)$. Its (infinitesimal) generator $\mathcal{A}$ is defined for all $f \in B\left(\mathbb{R}_{+}\right)$such that

$$
\mathcal{A} f=\lim _{t \rightarrow 0} \frac{P_{t} f-f}{t}
$$


exists in $B\left(\mathbb{R}_{+}\right)$. The domain of $\mathcal{A}$ is denoted by $\mathcal{D}(\mathcal{A})$. We write $B_{0}\left(\mathbb{R}_{+}\right)$for the set of functions $f$ in $B\left(\mathbb{R}_{+}\right)$for which $\lim _{t \rightarrow 0}\left\|P_{t} f-f\right\|=0$. Observe that

$$
(t, x) \mapsto P_{t} f(x)=\mathbb{E}_{x}\left[f\left(r_{t}\right)\right]
$$

is jointly measurable, for all $f \in B\left(\mathbb{R}_{+}\right)$. Indeed,

$$
(t, x) \mapsto \mathbb{E}_{x}\left[1_{\left[t_{1}, t_{2}\right]}(t) 1_{A}\right]=1_{\left[t_{1}, t_{2}\right]}(t) \mathbb{P}_{x}[A], \quad t_{1} \leq t_{2}, \quad A \in \mathcal{F},
$$

is jointly measurable. With regard to (A1) the claim follows by a monotone class argument. Hence the resolvent of $\mathcal{A}$, resp. $\left(P_{t}\right)$,

$$
R_{\lambda} f(x)=\int_{\mathbb{R}_{+}} e^{-\lambda t} P_{t} f(x) d t, \quad \lambda \in \mathbb{R}_{+}^{\circ},
$$

is well defined for all $f \in B\left(\mathbb{R}_{+}\right)$. It is well known that $R_{\lambda}$ is a one to one mapping from $B_{0}\left(\mathbb{R}_{+}\right)$onto $\mathcal{D}(\mathcal{A})$ with $R_{\lambda}=(\lambda-\mathcal{A})^{-1}$. Moreover $R_{\lambda}\left(B\left(\mathbb{R}_{+}\right)\right) \subset B_{0}\left(\mathbb{R}_{+}\right)$, see $[9$, Chapt. I.2].

We use the following notation, which differs from the terminology in [9].

Definition 2.1. A positive contraction semigroup $\left(T_{t}\right)$ on $B\left(\mathbb{R}_{+}\right)$is called a Feller semigroup if

$$
T_{t} f \in C_{0}\left(\mathbb{R}_{+}\right) \quad \text { and } \quad \lim _{t \rightarrow 0}\left\|T_{t} f-f\right\|=0, \quad \forall f \in C_{0}\left(\mathbb{R}_{+}\right) .
$$

The corresponding Markov process is called a Feller process.

For modelling purposes we will assume

(A2): Each $\mathbb{P}_{x}$ is a risk neutral measure, $x \in \mathbb{R}_{+}$; that is, any contingent claim $h \in L^{1}\left(\Omega, \mathcal{F}_{T}, \mathbb{P}_{x}\right)$ maturing at time $T$ has price

$$
\mathbb{E}_{x}\left[e^{-\int_{t}^{T} r_{s} d s} h \mid \mathcal{F}_{t}\right]
$$

at time $t \leq T$, given that $r_{0}=x$.

In particular, the price of a zero coupon bond is

$$
P(t, T)=\mathbb{E}_{x}\left[e^{-\int_{t}^{T} r_{s} d s} \mid \mathcal{F}_{t}\right]=\mathbb{E}_{x}\left[e^{-\int_{0}^{T-t} r_{s} d s} \circ \theta_{t} \mid \mathcal{F}_{t}\right]=\Pi\left(T-t, r_{t}\right)
$$

$\mathbb{P}_{x}$-a.s., where $\Pi(u, x):=\mathbb{E}_{x}\left[e^{-\int_{0}^{u} r_{s} d s}\right]$. By (A1) we have that $\Pi(u, x)$ is a strictly positive non-increasing continuous function in $u$.

\section{Affine Term Structure}

The simplest nontrivial relation (2) is exponential-affine.

Definition 3.1. A Markov short rate model $r$ as in Section 2 with

$$
\Pi(u, x)=e^{-A(u)-B(u) x},
$$

for some functions $A$ and $B$, is said to provide an affine term structure (ATS).

It is well know that property (2) excludes arbitrage possibilities in the bond market. And, clearly, it imposes structural properties on $A, B$ and $r$. It will turn out that $r$ is necessarily a very special type of Feller process and that $A$ and $B$ satisfy a system of generalized Riccati equations which are given by the infinitesimal generator of $r$. We already see from the preceding remarks that $A(u)$ and $B(u)$ a fortiori are nonnegative and non-decreasing continuous functions with 
$A(0)=B(0)=0$. Moreover, an immediate consequence of (2) is the following relation

$$
\begin{aligned}
e^{-A(T)-B(T) x} & =P(0, T)=\mathbb{E}_{x}\left[e^{-\int_{0}^{t} r_{s} d s} P(t, T)\right] \\
& =e^{-A(T-t)} \mathbb{E}_{x}\left[e^{-\int_{0}^{t} r_{s} d s} e^{-B(T-t) r_{t}}\right], \quad \forall t \leq T .
\end{aligned}
$$

Lemma 3.2. If $B\left(u^{*}\right)=0$ for some $u^{*} \in \mathbb{R}_{+}^{\circ}$, then $B \equiv 0$ and $A(u)=$ au for some $a \in \mathbb{R}_{+}$. Accordingly, the bond model is trivial, i.e. $P(t, T)=\exp (-a(T-t))$, and can be realized by the transition function

$$
p_{t}(x, d y)= \begin{cases}\delta_{a}(d y), & \text { if } t>0 \\ \delta_{x}(d y), & \text { if } t=0 .\end{cases}
$$

Proof. Let $u \in\left[0, u^{*}\right]$. Then $B(u)=0$ and, by relation (4),

$$
e^{(A(t+u)-A(u))-B(t+u) x}=e^{-A(t)-B(t) x}, \quad \forall t \in \mathbb{R}_{+} .
$$

Thus $B(t+u)=B(t)$ and $A(t+u)=A(t)+A(u)$ for all $t \in \mathbb{R}_{+}$and $u \in\left[0, u^{*}\right]$, and the lemma follows.

Consequently, we assume from now on that

$$
B(u)>0, \quad \forall u \in \mathbb{R}_{+}^{\circ} .
$$

Accordingly, the image of $B$

$$
\mathcal{I}:=\left\{B(u) \mid u \in \mathbb{R}_{+}\right\}
$$

contains an open interval, hence is a set of uniqueness for analytic functions in $\mathbb{R}_{+}^{\circ}$.

Definition 3.3. With regard to (1) we call

$$
Q_{t} f(x):=\mathbb{E}_{x}\left[e^{-\int_{0}^{t} r_{s} d s} f\left(r_{t}\right)\right], \quad f \in B\left(\mathbb{R}_{+}\right),
$$

the pricing semigroup.

The naming pricing semigroup will be legitimated in Proposition 3.4 below. Write $f_{\lambda}(x):=e^{-\lambda x}$ and define $\Lambda:=\left\{f_{\lambda} \mid \lambda \in \mathbb{R}_{+}^{\circ}\right\}$. By the Stone-Weierstrass theorem the linear hull $\mathcal{L}(\Lambda)$ of $\Lambda$ is dense in $C_{0}\left(\mathbb{R}_{+}\right)$. Equality (4) now reads, for $\lambda=B(u)$,

$$
Q_{t} f_{B(u)}(x)=e^{-(A(t+u)-A(u))-B(t+u) x} .
$$

This structural property actually holds for all $\lambda \in \mathbb{R}_{+}$.

Proposition 3.4. $\left(Q_{t}\right)$ is a Feller semigroup. Moreover, we have

$$
Q_{t} f_{\lambda}(x)=e^{-\rho(t, \lambda)-\sigma(t, \lambda) x}, \quad \forall t, \lambda, x \in \mathbb{R}_{+},
$$

for some functions $\rho(t, \lambda)$ and $\sigma(t, \lambda)$ which are analytic in $\lambda \in \mathbb{R}_{+}^{\circ}$, continuous in $\lambda \in \mathbb{R}_{+}$and in $t \in \mathbb{R}_{+}$, and satisfy

$$
\rho(t, B(u))=A(t+u)-A(u), \quad \sigma(t, B(u))=B(t+u) .
$$

Proof. Clearly $Q_{0} f=f$ and by the Markov property of $r$

$$
\begin{aligned}
Q_{t+s} f(x) & =\mathbb{E}_{x}\left[e^{-\int_{0}^{t+s} r_{u} d u} f\left(r_{t+s}\right)\right]=\mathbb{E}_{x}\left[e^{-\int_{0}^{t} r_{u} d u}\left(e^{-\int_{0}^{s} r_{u} d u} \circ \theta_{t}\right) f\left(r_{s} \circ \theta_{t}\right)\right] \\
& =\mathbb{E}_{x}\left[e^{-\int_{0}^{t} r_{u} d u} Q_{s} f\left(r_{t}\right)\right]=Q_{t} Q_{s} f(x), \quad \text { for all } f \in B\left(\mathbb{R}_{+}\right) .
\end{aligned}
$$

Moreover $0 \leq Q_{t} 1 \leq 1$, whence $\left(Q_{t}\right)$ is a positive contraction semigroup on $B\left(\mathbb{R}_{+}\right)$. 
Let $q_{t}(x, d y)$ denote the corresponding transition function, see [9, Chapt. II, Theorem 2.1]. For $t$ and $x$ fixed we have

$$
Q_{t} f_{\lambda}(x)=\int_{\mathbb{R}_{+}} e^{-\lambda y} q_{t}(x, d y)
$$

which is the Laplace transform of $q_{t}(x, d y)$ and hence analytic in $\lambda \in \mathbb{R}_{+}^{\circ}$. By (8) we have $Q_{t} f_{\lambda}(x)>0$ and

$$
Q_{t} f_{\lambda}(x) Q_{t} f_{\lambda}(y)=Q_{t} f_{\lambda}(0) Q_{t} f_{\lambda}(x+y), \quad \forall \lambda \in \mathcal{I} .
$$

But the product of two analytic functions is again analytic, hence equality (11) holds for all $\lambda \in \mathbb{R}_{+}$(notice that $0 \in \mathcal{I}$ ). Now fix $t, \lambda \in \mathbb{R}_{+}$and define $g(x):=$ $\frac{Q_{t} f_{\lambda}(x)}{Q_{t} f_{\lambda}(0)}$. The function $g$ is measurable, positive, bounded and satisfies the functional equation $g(x) g(y)=g(x+y)$. Hence there exist a nonnegative number $\sigma(t, \lambda)$ such that $g(x)=e^{-\sigma(t, \lambda) x}$, see $\left[1\right.$, Theorem 1.1.8.]. Clearly, we can write $Q_{t} f_{\lambda}(0)=$ $e^{-\rho(t, \lambda)}$ for some $\rho(t, \lambda) \in \mathbb{R}_{+}$, and thus (9) and (10) hold.

It remains to prove the Feller property of $\left(Q_{t}\right)$, or, equivalently, the continuity of $\rho(t, \lambda)$ and $\sigma(t, \lambda)$ in $t$. Fix $x \in \mathbb{R}_{+}$. First notice that every sequence $\left(q_{t_{n}}(x, d y)\right)$ with $t_{n} \rightarrow 0$ contains a subsequence $\left(q_{t_{n_{k}}}(x, d y)\right)$ which converges weakly to a measure $\mu(x, d y)$ on $\overline{\mathbb{R}}_{+}$. This implies

$$
\lim _{k \rightarrow \infty} Q_{t_{n_{k}}} f_{\lambda}(x)=\int_{\overline{\mathbb{R}}_{+}} e^{-\lambda y} \mu(x, d y)=\tilde{\mu}(x, \lambda), \quad \forall \lambda \in \mathbb{R}_{+}^{\circ}
$$

where $\tilde{\mu}(x, \lambda)$ denotes the Laplace transform of $\mu(x, d y)$. On the other hand

$$
\lim _{t \rightarrow 0} Q_{t} f_{\lambda}(x)=f_{\lambda}(x),
$$

for all $\lambda \in \mathcal{I}$, by continuity of $A$ and $B$. Uniqueness of the weak limit and analyticity of the Laplace transform yield $\tilde{\mu}(x, \lambda)=f_{\lambda}(x)$ and (12) holds for all $\lambda \in \mathbb{R}_{+}$.

Notice that $Q_{t} f_{\lambda^{\prime}} \leq Q_{t} f_{\lambda}$ for $\lambda^{\prime} \geq \lambda$ and $Q_{t} f_{\lambda} \in C_{0}\left(\mathbb{R}_{+}\right)$for $\lambda \in \mathcal{I} \backslash\{0\}$. From (12) it now follows easily that $\lim _{t \rightarrow 0}\left\|Q_{t} f_{\lambda}-f_{\lambda}\right\|=0$ for all $\lambda \in \mathbb{R}_{+}^{\circ}$. Since $\mathcal{L}(\Lambda)$ is dense in $C_{0}\left(\mathbb{R}_{+}\right)$, the proof is complete.

\section{CBI-Processes}

Feller semigroups with property (9) have been well studied. They appear as limits of Galton-Watson branching processes with immigration. We refer to [16] and [21] for background and further reference. Following their terminology we define

Definition 4.1. A Feller semigroup $\left(T_{t}\right)$ on $C_{0}\left(\mathbb{R}_{+}\right)$which satisfies

$$
T_{t} f_{\lambda}(x)=e^{-\phi(t, \lambda)-\psi(t, \lambda) x}, \quad \forall t, \lambda, x \in \mathbb{R}_{+},
$$

is called a CBI-semigroup. The Laplace exponents $\phi$ and $\psi$ are denoted as CBIexponents, and the corresponding Feller process as CBI-process (continuous state branching process with immigration).

Remark 4.2. In the notation of [16] this is in fact a stochastically continuous CBI-process.

Kawazu and Watanabe [16] provide a complete characterization of the generator of a CBI-semigroup $\left(T_{t}\right)$ and of its CBI-exponents $\phi(t, \lambda)$ and $\psi(t, \lambda)$. For the convenience of the reader we restate their main results and sketch the proofs. 
Theorem 4.3. Let $\left(T_{t}\right)$ be a CBI-semigroup with generator $\mathcal{G}$. Then $\mathcal{L}(\Lambda) \subset \mathcal{D}(\mathcal{G})$ and there exist real numbers

$$
\alpha \geq 0, \quad b \geq 0, \quad \beta, \quad c \geq 0, \quad \gamma \geq 0,
$$

and nonnegative Borel measures $m(d y)$ and $\mu(d y)$ on $\mathbb{R}_{+}^{\circ}$ satisfying

$$
\int_{\mathbb{R}_{+}^{o}}(1 \wedge y) m(d y)+\int_{\mathbb{R}_{+}^{o}}\left(1 \wedge y^{2}\right) \mu(d y)<\infty,
$$

such that for $f \in \mathcal{L}(\Lambda)$

$$
\begin{aligned}
\mathcal{G} f(x)= & \alpha x f^{\prime \prime}(x)+\left(b^{\prime}+\beta x\right) f^{\prime}(x)-(c+\gamma x) f(x) \\
& +\int_{\mathbb{R}_{+}^{\circ}}\left(f(x+y)-f(x)-f^{\prime}(x)(1 \wedge y)\right)(m(d y)+x \mu(d y)),
\end{aligned}
$$

where $b^{\prime}:=b+\int_{\mathbb{R}_{+}^{\circ}}(1 \wedge y) m(d y)$. Moreover, if we set

$$
\begin{aligned}
& R(\lambda):=-\alpha \lambda^{2}+\beta \lambda+\gamma+\int_{\mathbb{R}_{+}^{\circ}}\left(1-e^{-\lambda y}-\lambda(1 \wedge y)\right) \mu(d y) \\
& F(\lambda):=c+b \lambda+\int_{\mathbb{R}_{+}^{\circ}}\left(1-e^{-\lambda y}\right) m(d y),
\end{aligned}
$$

then $\psi(t, \lambda)$ and $\phi(t, \lambda)$ solve, for $\lambda \in \mathbb{R}_{+}$,

$$
\begin{aligned}
\frac{\partial \psi}{\partial t}(t, \lambda) & =R(\psi(t, \lambda)), \quad \psi(0, \lambda)=\lambda \\
\phi(t, \lambda) & =\int_{0}^{t} F(\psi(s, \lambda)) d s .
\end{aligned}
$$

Sketch of proof. By the semigroup property of $\left(T_{t}\right)$ it follows that

$$
\begin{aligned}
& \phi(t+s, \lambda)=\phi(t, \lambda)+\phi(s, \psi(t, \lambda)), \quad \phi(0, \lambda)=0 \\
& \psi(t+s, \lambda)=\psi(s, \psi(t, \lambda)), \quad \psi(0, \lambda)=\lambda,
\end{aligned}
$$

for all $t, s, \lambda \in \mathbb{R}_{+}$. Write $\Psi$ for the positive cone of functions of the form

$$
\psi(\lambda)=c_{0}+c_{1} \lambda+\int_{\mathbb{R}_{+}^{\circ}}\left(1-e^{-\lambda y}\right) n(d y),
$$

with $c_{0}, c_{1} \in \mathbb{R}_{+}$and $n(d y)$ a nonnegative Borel measure on $\mathbb{R}_{+}^{\circ}$ integrating $1 \wedge y$. Then $\Psi$ is closed under composition and under pointwise convergence on any set of uniqueness for analytic functions. It is well know that $\psi \in \Psi$ if and only if $e^{-\psi}$ is the Laplace transform of an infinitely divisible probability measure on $\overline{\mathbb{R}}_{+}$.

Using these facts and (20), one shows that $\phi(t, \lambda)$ is differentiable in $t \in \mathbb{R}_{+}$and (19) holds for some $F \in \Psi$, which is just (17).

Now write $X=\left(X_{t}\right)$ for the Feller process corresponding to $\left(T_{t}\right)$. Without loss of generality we assume that $X$ is cadlag in $\overline{\mathbb{R}}_{+}$, see $[18$, Theorem 2.7 , Chapt. III]. We have

$$
f_{\lambda}\left(X_{t}\right)=F_{\lambda}\left(u_{1}\left(X_{t}\right)\right),
$$

where $u_{1} \in \mathcal{D}(\mathcal{G}) \cap C^{\infty}\left(\mathbb{R}_{+}\right)$with $u_{1}^{\prime}(x)>0$, for all $x \in \mathbb{R}_{+}$, and $F_{\lambda}:=f_{\lambda} \circ u_{1}^{-1}$ has bounded first and second order derivatives for all $\lambda \in\left(\lambda_{0}, \infty\right)$, for some $\lambda_{0} \in \mathbb{R}_{+}$. In fact,

$$
u_{1}(x)=\int_{0}^{\bar{t}} T_{t} f_{\bar{\lambda}}(x) d t
$$


for some $\bar{t}, \bar{\lambda} \in \mathbb{R}_{+}^{\circ}$. Moreover, $u_{1}\left(X_{t}\right)-\int_{0}^{t} \mathcal{G} u_{1}\left(X_{s}\right) d s$ is a square integrable martingale. By Itô's formula, $T_{t} f_{\lambda}(x)=\mathbb{E}_{x}\left[F_{\lambda}\left(u_{1}\left(X_{t}\right)\right)\right]$ is of bounded variation in $t$, hence differentiable at $t=0$, for all $\lambda \in L_{0}$, where $L_{0}$ is a dense set in $\left(\lambda_{0}, \infty\right)$. Consequently, $R(\lambda)=\partial_{t} \psi(0, \lambda)$ exists and $f_{\lambda} \in \mathcal{D}(\mathcal{G}) \cap C_{0}^{2}\left(\mathbb{R}_{+}\right)$for $\lambda \in L_{0}$. Hence Lemma A.1 applies and one can write

$$
\mathcal{G} f_{\lambda}\left(x_{0}\right)=h\left(x_{0}, \lambda\right) e^{-\lambda x_{0}},
$$

where

$h\left(x_{0}, \lambda\right)=\alpha\left(x_{0}\right) \lambda^{2}+\beta\left(x_{0}\right) \lambda-\gamma\left(x_{0}\right)+\int_{\mathbb{R}_{+} \backslash\left\{x_{0}\right\}}\left(e^{-\lambda y}-1+\lambda \chi\left(y-x_{0}\right)\right) \nu\left(x_{0}, d y\right)$

with coefficients given by Lemma A.1. On the other hand

$$
\mathcal{G} f_{\lambda}\left(x_{0}\right)=\left.\frac{\partial}{\partial t} e^{-\phi(t, \lambda)-\psi(t, \lambda) x_{0}}\right|_{t=0}=\left(-x_{0} R(\lambda)-F(\lambda)\right) e^{-\lambda x_{0}} .
$$

One concludes $R(\lambda)=-h(1, \lambda)-F(\lambda)$ and finally derives (16) and (18), by analyticity of $\psi(t, \lambda)$ in $\lambda \in \mathbb{R}_{+}^{\circ}$. Equality (15) now follows easily by (22).

Remark 4.4. As a corollary we have that any conservative CBI-process $X$ is a semimartingale. Indeed, as shown in the preceding proof, $u_{1}(X)$ is a (cadlag) semimartingale. Moreover, $u_{1}:[0, \infty) \rightarrow\left(0, u_{1}(0)\right]$ is a diffeomorphism, which yields the assertion.

Remark 4.5. For any $f \in C_{c}^{2}\left(\mathbb{R}_{+}\right)$there exists a sequence $\left(f_{n}\right)$ in $\mathcal{L}(\Lambda)$, such that $f_{n} \rightarrow f, f_{n}^{\prime} \rightarrow f^{\prime}$ and $f_{n}^{\prime \prime} \rightarrow f^{\prime \prime}$ uniformly on $\mathbb{R}_{+}$. To see this, consider $\tilde{f}(y):=f\left(\log \left(y^{-1}\right)\right)$. Clearly, $\tilde{f} \in C^{2}([0,1])$. A version of the Stone-Weierstrass approximation theorem yields a sequence of polynomials $\left(p_{n}\right)$ which converges in the above sense towards $\tilde{f}$ on $[0,1]$, see [4, Section II.4]. Now set $f_{n}(x)=p_{n}\left(e^{-x}\right)$ to get the desired sequence.

Consequently, we have $\left\|\mathcal{G} f_{n}-\mathcal{G} f\right\| \rightarrow 0$, where $\mathcal{G} f \in C_{0}\left(\mathbb{R}_{+}\right)$is defined pointwise by (15). By the closedness of $\mathcal{G}$ we conclude that $C_{c}^{2}\left(\mathbb{R}_{+}\right) \subset \mathcal{D}(\mathcal{G})$ and (15) holds also for $f \in C_{c}^{2}\left(\mathbb{R}_{+}\right)$.

Conversely, we have

Theorem 4.6. Let $\alpha, b, \beta, c$ and $\gamma$ be real numbers and $m(d y), \mu(d y)$ nonnegative Borel measures on $\mathbb{R}_{+}^{\circ}$ such that (13) and (14) hold. Then $\mathcal{G}$, given by (15) on $\mathcal{L}(\Lambda)$, extends uniquely to the generator of a positive contraction semigroup $\left(T_{t}\right)$ on $B\left(\mathbb{R}_{+}\right)$. In fact, $\left(T_{t}\right)$ is a CBI-semigroup with CBI-exponents given by (18) and (19).

Sketch of proof. Note that $R(\lambda)$ is analytic in $\lambda \in \mathbb{R}_{+}^{\circ}$ and continuous in $\lambda \in \mathbb{R}_{+}$ with $R(0)=\gamma \geq 0$. Differentiating (16) gives, for $\lambda \in \mathbb{R}_{+}^{\circ}$,

$$
\begin{aligned}
& R^{\prime}(\lambda)=-2 \alpha \lambda+\beta+\int_{\mathbb{R}_{+}^{\circ}}\left(y e^{-\lambda y}-(1 \wedge y)\right) \mu(d y) \\
& R^{\prime \prime}(\lambda)=-2 \alpha-\int_{\mathbb{R}_{+}^{\circ}} y^{2} e^{-\lambda y} \mu(d y) .
\end{aligned}
$$

Hence $R$ is concave. However, as we shall see below, $R(\lambda)$ may fail to be Lipschitz continuous in $\lambda=0$. Nevertheless, there exists a unique global solution $\psi(t, \lambda)$ to (18) for all $\lambda \in \mathbb{R}_{+}^{\circ}$. Moreover, $\psi(t, \lambda) \rightarrow \psi(t, 0)$ for $\lambda \rightarrow 0$, which still solves (18). The same applies for $\phi(t, \lambda)$ and (19). It has been shown in [22, Theorem 4], see also $[25$, p. 456$]$, that $\psi(t, \cdot) \in \Psi$, for all $t \in \mathbb{R}_{+}$. By approximating the integral 
in (17), also $\phi(t, \cdot) \in \Psi$. Hence, for any $t, x \in \mathbb{R}_{+}$, there exists a unique infinitely divisible probability measure $q_{t}(x, \cdot)$ on $\overline{\mathbb{R}}_{+}$with Laplace transform $e^{-\phi(t, \lambda)-\psi(t, \lambda) x}$. Uniqueness of $\phi$ and $\psi$ yields the flow properties (20) and (21), respectively. As in the proof of Proposition 3.4, it now follows that $\left(q_{t}(x, d y)\right)$ is the transition function of a Feller process on $\mathbb{R}_{+}$which in fact is a CBI-process with generator $\mathcal{G}$.

The CBI-semigroup property yields $T_{t}(\mathcal{L}(\Lambda)) \subset \mathcal{L}(\Lambda)$. Thus Lemma 4.7 below applies with $D=\mathcal{L}(\Lambda)$. Hence $\mathcal{L}(\Lambda)$ is a core for $\mathcal{G}$, and the proof is complete.

Lemma 4.7 (Uniqueness). Let $\left(T_{t}\right)$ and $\left(T_{t}^{\prime}\right)$ be two contraction semigroups on $B\left(\mathbb{R}_{+}\right)$with generators $\mathcal{G}$ and $\mathcal{G}^{\prime}$. Assume that $\mathcal{G}=\mathcal{G}^{\prime}$ on a set $D \subset \mathcal{D}(\mathcal{G}) \cap \mathcal{D}\left(\mathcal{G}^{\prime}\right)$ with $T_{t}^{\prime}(D) \subset D$. Then $\left(T_{t}\right)=\left(T_{t}^{\prime}\right)$ on the closure $\bar{D}$.

Proof. Take $g \in D$. Then $T_{s}\left(T_{t-s}^{\prime} g\right)$ is differentiable in $s \in(0, t)$. It is easily seen that $\frac{d}{d s} T_{s}\left(T_{t-s}^{\prime} g\right)=T_{s}\left(\mathcal{G} T_{t-s}^{\prime} g\right)-T_{s}\left(\mathcal{G}^{\prime} T_{t-s}^{\prime} g\right)=0$. Hence by continuity $T_{s}\left(T_{t-s}^{\prime} g\right)=T_{t}^{\prime} g$ for all $s \leq t$ and thus $T_{t} g=T_{t}^{\prime} g$.

Remark 4.8. The proof of Theorem 4.6 shows that any CBI-process has a transition function of the form

$$
q_{t}(x, d y)=n_{t}(d y) * \nu_{t}(x, d y),
$$

where $n_{t}(d y)$ and $\nu_{t}(x, d y)$ are infinitely divisible probability measures on $\overline{\mathbb{R}}_{+}$and correspond to the Laplace transforms $e^{-\phi(t, \lambda)}$ and $e^{-\psi(t, \lambda) x}$, respectively. In fact, $\left(\nu_{t}(x, d y)\right)_{x \in \mathbb{R}_{+}}$is a continuous convolution semigroup on $\overline{\mathbb{R}}_{+}$. In the discrete time analog this factorization of $q_{t}(x, d y)$ no longer holds, see [13].

Shiga and Watanabe [21] actually showed the following stronger result. Let $D\left(\mathbb{R}_{+}\right)$be the subset of $\Omega$ consisting of all cadlag paths. Denote by $\left(\mathbb{Q}_{x}\right)_{x \in \mathbb{R}_{+}}$a family of probability measures on $D\left(\mathbb{R}_{+}\right)$and define

Definition 4.9. The family $\left(\mathbb{Q}_{x}\right)_{x \in \mathbb{R}_{+}}$is called infinitely decomposable if for all $n \in \mathbb{N}$ there exists a family $\left(\mathbb{Q}_{x}^{(n)}\right)_{x \in \mathbb{R}_{+}}$of probability measures on $D\left(\mathbb{R}_{+}\right)$satisfying

$$
\mathbb{Q}_{x_{1}+\cdots+x_{n}}=\mathbb{Q}_{x_{1}}^{(n)} * \cdots * \mathbb{Q}_{x_{n}}^{(n)}, \quad \forall x_{i} \in \mathbb{R}_{+} .
$$

If $\left(\mathbb{Q}_{x}\right)_{x \in \mathbb{R}_{+}}$corresponds to a Markov process, we call the Markov process itself infinitely decomposable.

Theorem 4.10. Every infinitely decomposable homogeneous Markov process having the strong Markov property and cadlag paths is a conservative CBI-process and vice versa.

Finally, we shall give a characterization of the conservative CBI-processes. Let $R(\lambda)$ be as in Theorem 4.3. By monotone convergence, see (23),

$$
\lim _{\lambda \downarrow 0} R^{\prime}(\lambda)=\beta+\int_{(1, \infty)}(y-1) \mu(d y) \leq+\infty .
$$

Hence we see that $R^{\prime}(0)=\lim _{\lambda \downarrow 0} R^{\prime}(\lambda)$ exists if and only if $\int_{(1, \infty)} y \mu(d y)<\infty$. In that case $R(\lambda)$ is Lipschitz continuous also at $\lambda=0$ and equation (18) has a unique global solution $\psi(t, \lambda)$ for all $\lambda \in \mathbb{R}_{+}$. If $R(\lambda)$ is not Lipschitz continuous at $\lambda=0$ then necessarily the right hand side of the equality in (24) equals $+\infty$. Accordingly, there exists $\lambda^{*} \in(0, \infty]$ such that $R(\lambda)>0$ for $\lambda \in\left(0, \lambda^{*}\right)$.

We now have the following result, see [16, Theorem 1.2]. 
Theorem 4.11. The CBI-semigroup $\left(T_{t}\right)$ from Theorem 4.3 is conservative if and only if $\gamma=c=0$ and

$$
\int_{0}^{\lambda^{*} \wedge 1} \frac{1}{R^{+}(\lambda)} d \lambda=\infty
$$

where $\lambda^{*}:=\inf \{\lambda>0 \mid R(\lambda)=0\} \in(0, \infty]$.

Proof. Notice that $\left(T_{t}\right)$ is conservative if and only if $\psi(t, 0)=\phi(t, 0) \equiv 0$.

If $R(\lambda)$ is Lipschitz continuous at $\lambda=0$ then (25) is trivially true, and it is clear by the preceding remarks that the theorem holds in that case.

Hence in the remainder of the proof we assume that $R(\lambda)$ is not Lipschitz continuous at $\lambda=0$. By the preceding discussion we then have $R(\lambda)>0$ for all $\lambda \in\left(0, \lambda^{*}\right)$.

Suppose first that $\left(T_{t}\right)$ is conservative. Since any solution $u(t)$ of (18) with $u(0)=0$ satisfies $u^{\prime}(0)=\gamma$ we must have $\gamma=0$, since otherwise $\psi(t, 0)>0$ for $t \in \mathbb{R}_{+}^{\circ}$. Similarly, we derive $c=0$. Denote by $u_{m}(t)$ the maximal solution of (18) with $u_{m}(0)=0$. We claim that $u_{m} \equiv 0$ if and only if (25) holds. Indeed, if $u_{m}(t)>0$ for some $t \in \mathbb{R}_{+}^{\circ}$ then, by its very definition, $u_{m}(t)>0$ for all $t \in \mathbb{R}_{+}^{\circ}$. Consequently

$$
t_{2}-t_{1}=\int_{t_{1}}^{t_{2}} \frac{u_{m}^{\prime}(t)}{R\left(u_{m}(t)\right)} d t=\int_{u_{m}\left(t_{1}\right)}^{u_{m}\left(t_{2}\right)} \frac{1}{R(\lambda)} d \lambda, \quad \forall 0<t_{1} \leq t_{2} .
$$

Since $u_{m}\left(t_{1}\right) \rightarrow 0$ for $t_{1} \rightarrow 0$, and $u_{m}\left(t_{2}\right) \rightarrow \lambda^{*}$ for $t_{2} \rightarrow \infty$, we conclude that the left hand side of (25) is finite. On the other hand, if the left hand side of (25) is finite, then

$$
\int_{0}^{u(t)} \frac{1}{R(\lambda)} d \lambda=t
$$

defines a local non-zero solution $u(t)$ of (18). Whence $u_{m}(t)>0$ for $t \in \mathbb{R}_{+}^{\circ}$.

Now assume that $u_{m}(t)>0$ for $t \in \mathbb{R}_{+}^{\circ}$. By uniqueness of $\psi(t, \lambda)$ for $\lambda \in \mathbb{R}_{+}^{\circ}$ we have, for any $s, t \in \mathbb{R}_{+}^{\circ}, u_{m}(t+s)=\psi\left(t, u_{m}(s)\right)$. But $u_{m}(s) \rightarrow 0$ for $s \rightarrow 0$, hence $\psi\left(t, u_{m}(s)\right) \rightarrow \psi(t, 0)=u_{m}(t)>0$, which is impossible.

Conversely, suppose that $c=\gamma=0$ and that (25) holds. As shown above we then have $u_{m} \equiv 0$. This in turn implies $\psi(t, 0)=\phi(t, 0) \equiv 0$. Hence $\left(T_{t}\right)$ is conservative.

Remark 4.12. It is clear that $R(\lambda)$ is Lipschitz continuous in $\lambda$ if and only if so is

$$
G(\lambda):=\int_{\mathbb{R}_{+}^{\circ}}\left(1-e^{-\lambda y}-\lambda(1 \wedge y)\right) \mu(d y) .
$$

Moreover, if $G(\lambda)$ is not Lipschitz continuous in $\lambda=0$, then it is not hard to see that

$$
\frac{1}{2} G^{+}(\lambda) \leq R^{+}(\lambda) \leq 2 G^{+}(\lambda),
$$

for $\lambda$ small enough. Hence Theorem 4.11 remains true if one replaces (25) by the condition

$$
\int_{0}^{\tilde{\lambda} \wedge 1} \frac{1}{G^{+}(\lambda)} d \lambda=\infty
$$

with $\tilde{\lambda}:=\inf \{\lambda>0 \mid G(\lambda)=0\}$, which is a property of $\mu(d y)$ only. 


\section{The Short Rates as CBI-Process}

So far we have shown that, if $r$ induces an ATS, the pricing semigroup $\left(Q_{t}\right)$ given by (7) is necessarily a CBI-semigroup. Its generator $\mathcal{B}$ accordingly is given by the right hand side of (15), for some coefficients $\alpha, b, \beta, c, \gamma, m(d y)$ and $\mu(d y)$ as in Theorem 4.3. We shall prove that also $r$ has to be a CBI-process in this case. Therefore we apply the Feynman-Kac formula and characterize the generator $\mathcal{A}$ of $\left(P_{t}\right)$, the semigroup related to $r$.

Introduce the unbounded closed operator $V$ on $B\left(\mathbb{R}_{+}\right)$by $V f(x):=x f(x)$. We denote the resolvent of $\mathcal{B}$ by $R_{\lambda}^{\mathcal{B}}$. Observe that

$$
R_{\lambda}^{\mathcal{B}} f(x)=\int_{\mathbb{R}_{+}} e^{-\lambda t} Q_{t} f(x) d t
$$

is well defined for any $f \in B\left(\mathbb{R}_{+}\right)$, since $\left(Q_{t}\right)$ is Feller. We shall use two versions of the Feynman-Kac formula.

Lemma 5.1 (Feynman-Kac I).

$$
R_{\lambda}^{\mathcal{B}} g=R_{\lambda}\left(g-V\left(R_{\lambda}^{\mathcal{B}} g\right)\right)
$$

for all $g \in B\left(\mathbb{R}_{+}\right)$with $R_{\lambda}^{\mathcal{B}} g \in \mathcal{D}(V)$.

Proof. It is enough to consider the case $g \geq 0$. Now proceed as in [19, p. 273], taking into account (A1).

We can now prove the following particular version.

Lemma 5.2 (Feynman-Kac II). We have $\mathcal{L}(\Lambda) \subset \mathcal{D}(\mathcal{B}) \cap \mathcal{D}(\mathcal{A}) \cap \mathcal{D}(V)$ and

$$
\mathcal{B} f=\mathcal{A} f-V f
$$

for $f \in \mathcal{L}(\Lambda)$.

Proof. From Theorem 4.3 we know that $\mathcal{L}(\Lambda) \subset \mathcal{D}(\mathcal{B})$. Moreover, it is clear that $\mathcal{L}(\Lambda) \subset \mathcal{D}(V)$. It remains to show $\mathcal{L}(\Lambda) \subset \mathcal{D}(\mathcal{A})$.

Let $f \in \mathcal{L}(\Lambda)$. Then there exists a unique $g \in C_{0}\left(\mathbb{R}_{+}\right)$with $R_{\lambda}^{\mathcal{B}} g=f$. Since $R_{\lambda}^{\mathcal{B}} g \in \mathcal{D}(V)$ Lemma 5.1 applies and $f=R_{\lambda}(g-V f) \in R_{\lambda}\left(B\left(\mathbb{R}_{+}\right)\right) \subset B_{0}\left(\mathbb{R}_{+}\right)$. Thus $\mathcal{L}(\Lambda) \subset B_{0}\left(\mathbb{R}_{+}\right)$. Since $B_{0}\left(\mathbb{R}_{+}\right)$is closed therefore

$$
C_{0}\left(\mathbb{R}_{+}\right)=\overline{\mathcal{L}(\Lambda)} \subset B_{0}\left(\mathbb{R}_{+}\right)
$$

Consequently, we have $R_{\lambda}\left(C_{0}\left(\mathbb{R}_{+}\right)\right) \subset R_{\lambda}\left(B_{0}\left(\mathbb{R}_{+}\right)\right)=\mathcal{D}(\mathcal{A})$. Take $f$ and $g$ as above. Clearly $g-V f \in C_{0}\left(\mathbb{R}_{+}\right)$, whence $f=R_{\lambda}(g-V f) \in \mathcal{D}(\mathcal{A})$. We have thus shown that $\mathcal{L}(\Lambda) \subset \mathcal{D}(\mathcal{A})$.

Let $f$ and $g$ be as above. From Lemma 5.1 we have $R_{\lambda} g=f+R_{\lambda}(V f)$. Since $f \in \mathcal{D}(\mathcal{A})$ and $V f \in C_{0}\left(\mathbb{R}_{+}\right) \subset B_{0}\left(\mathbb{R}_{+}\right)$we can solve this equation and get $g=$ $(\lambda-\mathcal{A}) f+V f$. On the other hand $g=(\lambda-\mathcal{B}) f$. We conclude

$$
(\lambda-\mathcal{B}) f=(\lambda-\mathcal{A}) f+V f
$$

and the lemma follows.

As a consequence of Lemma 5.2, the generator $\mathcal{A}$ of $\left(P_{t}\right)$ can be represented on $\mathcal{L}(\Lambda)$ by the right hand side of $(15)$ with $\gamma$ replaced by $\tilde{\gamma}=\gamma-1$. It is not a priori clear whether $\tilde{\gamma}$ is negative or not. However, apply the positive maximum principle (see e.g. [18, Proposition 1.5, Chapter VII]) to $\mathcal{A} f$ with $f(x)=e^{-\lambda x}-e^{-2 \lambda x} \in$ $\mathcal{D}(\mathcal{A})$, and let $\lambda$ tend to zero to see that $\tilde{\gamma}<0$ is impossible. Theorem 4.6 now 
yields that $r$ is a CBI-process. Since $r$ is conservative we have that $c=\tilde{\gamma}=0$ and $\mu(d y)$ satisfies (26), see Theorem 4.11.

We arrive at our main result.

Theorem 5.3 (Main theorem). Let $r$ be a homogeneous Markov process in $\mathbb{R}_{+}$as given in Section 2, satisfying:

(A1): The process $r$ is progressively measurable and

$$
\int_{0}^{t} r_{s} d s<\infty, \quad \mathbb{P}_{x} \text {-a.s. } \forall t \in \mathbb{R}_{+} .
$$

(A2): Each $\mathbb{P}_{x}$ is a risk neutral measure, $x \in \mathbb{R}_{+}$, see (1).

Then the following four conditions are equivalent:

i) $r$ provides a non-trivial ATS, see (6).

ii) $r$ is a CBI-process, uniquely characterized by its generator on $\mathcal{L}(\Lambda) \cup C_{c}^{2}\left(\mathbb{R}_{+}\right)$

$$
\begin{aligned}
\mathcal{A} f(x)= & \alpha x f^{\prime \prime}(x)+\left(b^{\prime}+\beta x\right) f^{\prime}(x) \\
& +\int_{\mathbb{R}_{+}^{\circ}}\left(f(x+y)-f(x)-f^{\prime}(x)(1 \wedge y)\right)(m(d y)+x \mu(d y)),
\end{aligned}
$$

where $b^{\prime}:=b+\int_{\mathbb{R}_{+}^{o}}(1 \wedge y) m(d y)$, for some numbers $\alpha, b \in \mathbb{R}_{+}, \beta \in \mathbb{R}$ and nonnegative Borel measures $m(d y)$ and $\mu(d y)$ on $\mathbb{R}_{+}^{\circ}$ satisfying (14) and (26).

iii) The pricing semigroup $\left(Q_{t}\right)$, given by (7), is a CBI-semigroup whose $C B I$ exponents $\rho(t, \lambda)$ and $\sigma(t, \lambda)$ satisfy the system of equations, for $\lambda \in \mathbb{R}_{+}$,

$$
\begin{aligned}
\frac{\partial \sigma}{\partial t}(t, \lambda) & =R(\sigma(t, \lambda)), \quad \sigma(0, \lambda)=\lambda \\
\rho(t, \lambda) & =\int_{0}^{t} F(\sigma(s, \lambda)) d s
\end{aligned}
$$

where

$$
\begin{aligned}
& R(\lambda):=-\alpha \lambda^{2}+\beta \lambda+1+\int_{\mathbb{R}_{+}^{\circ}}\left(1-e^{-\lambda y}-\lambda(1 \wedge y)\right) \mu(d y) \\
& F(\lambda):=b \lambda+\int_{\mathbb{R}_{+}^{\circ}}\left(1-e^{-\lambda y}\right) m(d y)
\end{aligned}
$$

with $\alpha, b, \beta, m(d y)$ and $\mu(d y)$ as in condition ii).

iv) $r$ is an infinitely decomposable Feller process.

Proof. Only the implication ii) $\Rightarrow$ iii) remains to be proved. Again we will use the Feynman-Kac formula to identify the generator $\mathcal{B}$ of $\left(Q_{t}\right)$. Let $R_{\lambda}^{\mathcal{B}}$ denote the resolvent of $\mathcal{B}$. Analogously to Lemma 5.1 one shows

$$
R_{\lambda} g=R_{\lambda}^{\mathcal{B}}\left(g+V\left(R_{\lambda} g\right)\right)
$$

for all $g \in B\left(\mathbb{R}_{+}\right)$with $R_{\lambda} g \in \mathcal{D}(V)$. If one exchanges the roles of $\mathcal{A}$ and $\mathcal{B}$ in the proof of Lemma 5.2 and uses (32) instead of Lemma 5.1 one gets that $\mathcal{L}(\Lambda) \subset \mathcal{D}(\mathcal{B})$ and

$$
\mathcal{B} f=\mathcal{A} f-V f, \quad \text { for } f \in \mathcal{L}(\Lambda) .
$$

Hence $\left(Q_{t}\right)$ is a CBI-semigroup and the assertion follows. 
The functions $A(u)$ and $B(u)$ appearing in the term structure (3) are given by $A(u)=\rho(u, 0)$ and $B(u)=\sigma(u, 0)$, see (10). Hence condition iii) of Theorem 5.3 yields

Corollary 5.4. The functions $A$ and $B$ solve uniquely the generalized Riccati equations

$$
\begin{aligned}
B^{\prime}(u) & =R(B(u)), \quad B(0)=0 \\
A(u) & =\int_{0}^{u} F(B(s)) d s,
\end{aligned}
$$

where $R$ and $F$ are defined in (30) and (31).

Proof. Only the uniqueness has to be verified. But any solution $u(t)$ of $(28)$ with $u(0)=0$ satisfies $u(t)>0$ for $t \in \mathbb{R}_{+}^{\circ}$, since $u^{\prime}(0)=1$. Hence, by uniqueness of $\sigma(t, \lambda)$ for $\lambda \in \mathbb{R}_{+}^{\circ}$, we have $u(t+s)=\sigma(t, u(s))$ for all $t, s \in \mathbb{R}_{+}^{\circ}$. Letting $s \rightarrow 0$ we conclude $u(t)=\sigma(t, 0)$, which gives our assertion.

\section{Pricing a European Bond Option}

Here and subsequently, $r=\left(r_{t}\right)$ is a Markov short rate process satisfying the conditions of Theorem 5.3. Then the price today of a European claim $f\left(r_{T}\right), f \in$ $B\left(\mathbb{R}_{+}\right)$, due at time $T$, is given by the pricing semigroup

$$
Q_{T} f(x)=\int_{\mathbb{R}_{+}} f(y) q_{T}(x, d y),
$$

given that $r_{0}=x$, see (1). For

$$
f(x)=\left(e^{-A(S-T)-B(S-T) x}-K\right)^{+}=\left(e^{-A(S-T)-B(S-T) x}-K\right) 1_{\left[0, r^{*}\right]}(x),
$$

with

$$
r^{*}:=(-\log (K)-A(S-T))^{+} / B(S-T),
$$

we get the price $\pi(x)$ of a European call option on a bond maturing at time $S$ with strike price $K$ and expiry date $T \leq S$. Let $U \geq T$ and recall the definition of the $U$-forward measure

see e.g. [17]. It then follows that

$$
\frac{d \mathbb{P}_{x}^{U}}{d \mathbb{P}_{x}}=\frac{e^{-\int_{0}^{U} r_{s} d s}}{e^{-A(U)-B(U) x}},
$$

$$
q_{T}^{U}(x, d y):=e^{A(U)-A(U-T)+B(U) x} e^{-B(U-T) y} q_{T}(x, d y)
$$

is the distribution of $r_{T}$ under $\mathbb{P}_{x}^{U}$, and the Laplace transform of $q_{T}^{U}(x, d y)$ is given by

$$
\int_{\mathbb{R}_{+}} e^{-\lambda y} q_{T}^{U}(x, d y)=e^{A(U)-A(U-T)-\rho(T, \lambda+B(U-T))} e^{-(\sigma(T, \lambda+B(U-T))-B(U)) x} .
$$

We now can write

$$
\pi(x)=e^{-A(S)-B(S) x} q_{T}^{S}\left(x,\left[0, r^{*}\right]\right)-K e^{-A(T)-B(T) x} q_{T}^{T}\left(x,\left[0, r^{*}\right]\right) .
$$

Thus, if we can invert the Laplace transform (34) analytically, we get a closed form expression for $\pi(x)$ by (35).

Remark 6.1. Given that $\rho(t, \lambda)$ and $\sigma(t, \lambda)$ are analytic at $\lambda=0$, one can use saddle-point approximations to compute numerically the tail probabilities appearing in (35). See Rogers and Zane [20] for that approach. 
Similarly, one derives the analogous expression for the price of a put option. That way, one can price any cap, floor and swaption within the present model.

\section{An Example: CIR With Jumps}

We shall now derive an extension of the CIR model including jumps which still allows for a closed form expression (35). We choose the parameters according to Theorem 5.3 as follows:

$$
\alpha, b, \beta \in \mathbb{R}_{+}, \quad m(d y)=c e^{-d y} d y, \quad \mu=0,
$$

for some $c, d \in \mathbb{R}_{+}, d>0$, and let the generator of $r$ be given by

$$
\mathcal{A} f(x)=\alpha x f^{\prime \prime}(x)+(b-\beta x) f^{\prime}(x)+c \int_{\mathbb{R}_{+}^{\circ}}(f(x+y)-f(x)) e^{-d y} d y,
$$

see (27). Thus $r$ can be realized as a jump-diffusion process whose (positive) jump size and inter-arrival times are exponentially distributed with parameters $d$ and $c / d$, respectively, see e.g. [10, p. 163]. Now (30) and (31) become

$$
\begin{aligned}
& R(\lambda)=-\alpha \lambda^{2}-\beta \lambda+1 \\
& F(\lambda)=b \lambda+\frac{c \lambda}{d \lambda+d^{2}} .
\end{aligned}
$$

The solutions $\sigma$ and $\rho$ of (28) and (29) are given subsequently. Write $\gamma:=\sqrt{\beta^{2}+4 \alpha}$. The following singularities have to be treated separately, namely $\alpha=0$ and $d=$ $d_{0}:=(\beta+\gamma) /(2 \alpha)$. We shall discuss the cases in the order i) $\alpha>0$ and $d<d_{0}$, ii) $\alpha>0$ and $d>d_{0}$, iii) $\alpha>0$ and $d=d_{0}$, and finally iv) $\alpha=0$.

We remark that the present process $r$ has already been introduced by Duffie and Gârleanu [6] for financial applications. They also derived the equations (28) and (29) and the general solutions $\sigma$ and $\rho$, given in (36) and (37) below.

Case $i$ ): $\alpha>0$ and $d<d_{0}$. We have

$$
\begin{aligned}
\sigma(t, \lambda)= & \frac{L_{1}(t) \lambda+L_{2}(t)}{L_{3}(t) \lambda+L_{4}(t)} \\
\rho(t, \lambda)= & -\frac{b}{\alpha} \log \left(\frac{L_{5}(t)}{L_{3}(t) \lambda+L_{4}(t)}\right) \\
& -\frac{c}{1+\beta d-\alpha d^{2}} \log \left(e^{-\frac{t}{d}} \frac{\left(L_{1}(t)+d L_{3}(t)\right) \lambda+L_{2}(t)+d L_{4}(t)}{L_{5}(t)(\lambda+d)}\right),
\end{aligned}
$$

where

$$
\begin{aligned}
& L_{1}(t):=\gamma\left(e^{\gamma t}+1\right)-\beta\left(e^{\gamma t}-1\right) \\
& L_{2}(t):=2\left(e^{\gamma t}-1\right) \\
& L_{3}(t):=2 \alpha\left(e^{\gamma t}-1\right) \\
& L_{4}(t):=\gamma\left(e^{\gamma t}+1\right)+\beta\left(e^{\gamma t}-1\right) \\
& L_{5}(t):=2 \gamma e^{(\gamma+\beta) t / 2} .
\end{aligned}
$$


Having in mind that $A(t)=\sigma(t, 0)$ and $B(t)=\rho(t, 0)$, one computes the right hand side of (34)

$$
\begin{aligned}
\int_{\mathbb{R}_{+}} e^{-\lambda y} q_{T}^{U}(x, d y)= & \left(C_{1}(T, U)\left(C_{2}(T, U)+\frac{1}{C_{3}(T, U) \lambda+1}\right)\right)^{\frac{c}{1+\beta d-\alpha d^{2}}} \\
& \times \frac{e^{-C_{5}(T, U) x+\frac{C_{5}(T, U) x}{C_{4}(T, U) \lambda+1}}}{\left(C_{4}(T, U) \lambda+1\right)^{\frac{b}{\alpha}}}
\end{aligned}
$$

where

$$
\begin{aligned}
& C_{1}(T, U):=\left(\frac{1+\beta d-\alpha d^{2}}{2 \gamma}\right) \frac{L_{2}(T) L_{4}(U-T)}{L_{2}(U)+d L_{4}(U)} \\
& C_{2}(T, U):=\left(\frac{1}{1+\beta d-\alpha d^{2}}\right) \frac{\left(L_{1}(T)+d L_{3}(T)\right)\left(L_{2}(U-T)+d L_{4}(U-T)\right)}{L_{2}(T) L_{4}(U-T)} \\
& C_{3}(T, U):=\frac{L_{4}(U-T)}{L_{2}(U-T)+d L_{4}(U-T)} \\
& C_{4}(T, U):=\frac{L_{3}(T) L_{4}(U-T)}{2 \gamma L_{4}(U)} \\
& C_{5}(T, U):=\frac{L_{1}(T)}{L_{3}(T)}-\frac{L_{2}(U)}{L_{4}(U)} .
\end{aligned}
$$

Notice that $c=0$ just yields the classical CIR model. Indeed, the second factor in (38) is, up to scaling by $1 / C_{4}(T, U)$, the Laplace transform of a noncentral chisquare distribution with $2 b / \alpha$ degrees of freedom and parameter of noncentrality $2 C_{5}(T, U) x$. The density is therefore given by $f_{2 b / \alpha, 2 C_{5}(U, U) x}\left(y / C_{4}(T, U)\right)$, where

$$
f_{2 \delta, 2 \zeta}(y):=e^{-\zeta-y} \sqrt{(y / \zeta)^{\delta-1}} I_{\delta-1}(2 \sqrt{\zeta y}), \quad \delta, \zeta \in \mathbb{R}_{+}^{\circ} .
$$

Here $I_{\nu}$ is the modified Bessel function of the first kind of order $\nu>-1$, see [11, Example XIII,3(e)] and also [15] for a description of the noncentral chi-square distribution. That way we get the well-known formula for $\pi(x)$ which was first derived by Cox, Ingersoll and Ross in [5].

If $c>0$ then (38) is the Laplace transform of the convolution of a infinitely divisible distribution with the above noncentral chi-square distribution. In particular, if we choose

$$
\frac{c}{1+\beta d-\alpha d^{2}}=n \in \mathbb{N}
$$

then the first factor in (38) can be expanded

$$
C_{1}(T, U)^{n} \sum_{k=0}^{n}\left(\begin{array}{l}
n \\
k
\end{array}\right) C_{2}(T, U)^{n-k}\left(\frac{1}{C_{3}(T, U) \lambda+1}\right)^{k} .
$$

This is the Laplace transform of a mixture of gamma distributions with the density

$$
C_{1}(T, U)^{n} \sum_{k=0}^{n}\left(\begin{array}{l}
n \\
k
\end{array}\right) C_{2}(T, U)^{n-k} g_{1 / C_{3}(T, U), k}(y),
$$

where

$$
g_{\rho, \nu}(y)=\frac{1}{\Gamma(\nu)} \rho^{\nu} y^{\nu-1} e^{-\rho y}, \quad \rho, \nu \in \mathbb{R}_{+}^{\circ},
$$

is the gamma density, and we set $g_{\rho, 0}(y) d y=\delta_{0}$, the Dirac measure in 0 . Hence we arrive at a closed form expression for the pricing formula (35) consisting of 
a finite sum of convolutions of gamma distributions with a noncentral chi-square distribution.

Case ii): $\alpha>0$ and $d>d_{0}$ leads to a similar result. Only the first factor in (38) has to be replaced by its inverse.

Case iii): $\alpha>0$ and $d=d_{0}$. The second summand in (37) is replaced by a rational function in $\lambda$. Accordingly, the first factor in (38) becomes

$$
e^{-D_{1}(T, U)+\frac{D_{1}(T, U)}{D_{2}(T, U) \lambda+1}}
$$

for some positive numbers $D_{1}(T, U)$ and $D_{2}(T, U)$. We omit their explicit form. We only mention that there is, unfortunately, no useful relation between $D_{2}(T, U)$ and $C_{4}(T, U)$. Again, (41) corresponds to a mixture of gamma distributions. However, this time involving an infinite sum, see [11, Example XIII,3(c)].

Case $i v): \alpha=0$. The dynamics of $r$ are reduced to a drift term plus positive jumps. On the other hand we get an extremely simple expression for the pricing formula (35). Set

$$
\begin{aligned}
L_{6}(t) & :=\frac{1-e^{-\beta t}}{\beta} \\
L_{7}(t) & :=e^{\beta t}-1+\beta d e^{\beta t},
\end{aligned}
$$

then we have

$$
\begin{aligned}
& \sigma(t, \lambda)=e^{-\beta t} \lambda+L_{6}(t) \\
& \rho(t, \lambda)=b L_{6}(t) \lambda+\frac{b}{\beta}\left(t-L_{6}(t)\right)+\frac{c}{d} t+\frac{c}{1+\beta d} \log \left(\frac{\lambda+d}{\lambda+\left(d+L_{6}(t)\right) e^{\beta t}}\right) .
\end{aligned}
$$

The Laplace transform (38) becomes

$$
\begin{aligned}
\int_{\mathbb{R}_{+}} e^{-\lambda y} q_{T}^{U}(x, d y)= & \left(C_{6}(T, U)\left(C_{7}(T, U)+\frac{1}{C_{8}(T, U) \lambda+1}\right)\right)^{\frac{c}{1+\beta d}} \\
& \times e^{-\left(e^{-\beta T} x+b L_{6}(T)\right) \lambda},
\end{aligned}
$$

where

$$
\begin{aligned}
C_{6}(T, U) & :=\frac{L_{7}(U)-L_{7}(U-T)}{L_{7}(U)} \\
C_{7}(T, U) & :=\frac{L_{7}(U-T)}{L_{7}(U)-L_{7}(U-T)} \\
C_{8}(T, U) & :=\frac{\beta e^{\beta(U-T)}}{L_{7}(U-T)} .
\end{aligned}
$$

As in case i) we choose

$$
\frac{c}{1+\beta d}=n \in \mathbb{N} .
$$

Then the first factor in (42) is the Laplace transform of a mixture of gamma distributions with the density given by $(39)$, where $C_{1}(T, U)-C_{3}(T, U)$ is replaced by $C_{6}(T, U)-C_{8}(T, U)$, respectively. The second factor in (42) is the Laplace transform of the Dirac measure in the point $r_{d}(x, T):=e^{-\beta T} x+b L_{6}(T)$, which corresponds to the deterministic drift part in the dynamics of $r$, i.e.

$$
\partial_{t} r_{d}(x, t)=b-\beta r_{d}(x, t) .
$$


This would be the valued of $r_{t}$ if no jumps had occurred until time $t$. As a result we get

$$
q_{T}^{U}\left(x,\left[0, r^{*}\right]\right)=C_{6}(T, U)^{n} \sum_{k=0}^{n}\left(\begin{array}{l}
n \\
k
\end{array}\right) C_{7}(T, U)^{n-k} G_{1 / C_{8}(T, U), k}\left(\left(r^{*}-r_{d}(x, T)\right)^{+}\right),
$$

where $G_{\rho, \nu}$ denotes the cumulative distribution function of the gamma distribution with density (40). Note that $r^{*}>r_{d}(x, T)$ is equivalent to, see (33),

$$
K<e^{-A(S-T)-B(S-T) r_{d}(x, T)} .
$$

On the right hand side of (43) stands the time $T$ price of the $S$-bond computed for the "jump-less" short rate $r_{d}(x, T)$. Hence we see that $\pi(x)$ is non zero, see (35), only if that "jump-less bond" is in the money at expiry date $T$.

\section{The Multidimensional Case}

If the bond model is driven by $n \geq 2$ Markovian factors $X^{1}, \ldots, X^{n}$ we do not have such a strong result anymore. That is, affine term structure

$$
P(t, T)=e^{-A(T-t)-\sum_{i=1}^{n} B^{i}(T-t) X_{t}^{i}}
$$

does not imply the $n$-dimensional version of condition ii) of Theorem 5.3. This can be seen from the following example, where $n=2$.

Set $A=0, B^{1}(u)=u$ and $B^{2}(u)=u^{2} / 2$. It is not hard to see, compare e.g. with [12, Proposition 3.2], that any diffusion $X=\left(X^{1}, X^{2}\right)$ in $\mathbb{R}_{+} \times \mathbb{R}_{+}$of the form

$$
\begin{aligned}
& d X_{t}^{1}=X_{t}^{2} d t+\sqrt{\beta\left(X_{t}^{1}, X_{t}^{2}\right)} d W_{t} \\
& d X_{t}^{2}=\beta\left(X_{t}^{1}, X_{t}^{2}\right) d t
\end{aligned}
$$

provides an arbitrage-free ATS model

$$
P(t, T)=e^{-(T-t) X_{t}^{1}-\frac{(T-t)^{2}}{2} X_{t}^{2}} .
$$

Clearly, $\beta\left(x_{1}, x_{2}\right)$ has not to be affine in $\left(x_{1}, x_{2}\right)$ in order that SDE (44) has a strong solution in $\mathbb{R}_{+} \times \mathbb{R}_{+}$.

To see this, take $\beta\left(x_{1}, x_{2}\right)=\beta\left(x_{1}\right):=\left(1 \wedge x_{1}\right)^{+}$. Existence of a non-exploding continuous weak solution of SDE (44) in $\mathbb{R}^{2}$ follows by continuity and linear growth of the coefficients, see [14, Chapt. IV.2]. Proceeding as in the proof of [14, Theorem 3.2] we show pathwise uniqueness, implying existence of a unique strong solution in $\mathbb{R}^{2}$. Indeed, adapting their notation we can show that, whenever $X=\left(X^{1}, X^{2}\right)$ and $Y=\left(Y^{1}, Y^{2}\right)$ are two weak solutions (on the same filtered probability space and with the same Brownian motion) of SDE (44) with $X_{0}=Y_{0}$, then

$$
\mathbb{E}\left[\varphi_{n}\left(X_{t}^{1}-Y_{t}^{1}\right)+\left|X_{t}^{2}-Y_{t}^{2}\right|\right] \leq K \int_{0}^{t} \mathbb{E}\left[\left|X_{s}^{2}-Y_{s}^{2}\right|\right] d s+\frac{t}{n},
$$

where $K$ is a constant independent of $n$. By Gronwall's lemma $X_{t}^{2}=Y_{t}^{2}$ and, since $\varphi_{n}(x) \uparrow|x|$ as $n \rightarrow \infty$, also $X_{t}^{1}=Y_{t}^{1}$ for all $t \in \mathbb{R}_{+}$a.s.

It remains to check invariance of $\mathbb{R}_{+} \times \mathbb{R}_{+}$. Observe that $X_{t}^{2} \in \mathbb{R}_{+}$whenever $X_{0}^{2} \in \mathbb{R}_{+}$by positivity of $\beta$. Now let $X_{0} \in \mathbb{R}_{+} \times \mathbb{R}_{+}$. For $\delta, \epsilon>0$ set

$$
\tau_{\delta, \epsilon}:=\inf \left\{t \in \mathbb{R}_{+} \mid X_{t}^{1} \leq-\epsilon \text { and } X_{s}^{1}<0 \text { for all } s \in[t-\delta, t]\right\} .
$$


Then on $\left\{\tau_{\delta, \epsilon}<\infty\right\}$ we have

$$
0>X_{\tau_{\delta, \epsilon}}^{1}-X_{\tau_{\delta, \epsilon}-\delta}^{1}=\int_{\tau_{\delta, \epsilon}-\delta}^{\tau_{\delta, \epsilon}} X_{s}^{2} d s \geq 0,
$$

a contradiction. Hence $\tau_{\delta, \epsilon}=\infty$ a.s. and therefore $X_{t}^{1} \in \mathbb{R}_{+}$.

This example also shows that the assumptions of the proposition in [7] are really needed. Indeed, they have to suppose linear independence of the functions $B^{i}(u)$ and $B^{i}(u) B^{j}(u)$.

Conditions ii)-iv) of Theorem 5.3, however, remain equivalent also in higher dimensions, see [25]. Moreover, condition iii) implies ATS. From a practical point of view this is satisfactory, since it provides a tool for constructing multifactor ATS models.

\section{Conclusion}

We have given a complete characterization of all nonnegative time homogeneous Markov short rate processes which provide an ATS. These form exactly the class of conservative CBI-processes. We have gained new insight into the structure of the pricing formulas for interest rate sensitive European claims. A particular model which extends the classical CIR model by involving jumping short rates is presented. Within this model we have derived closed form expressions for the European type bond options.

An extension towards more general multifactor pricing models is possible and is actually work in progress. However, we have already seen in the previous section that there is no such strong characterization result for multifactor ATS models anymore.

\section{Appendix A. Infinitesimal Generators of Feller semigroups}

The following representation for infinitesimal generators of Feller semigroups goes back to [24], see also [23, Chapt. 9.4] for a detailed discussion.

Set $\chi(x):=\frac{x}{|x|}(1 \wedge|x|)$.

Lemma A.1. Let $\left(P_{t}\right)$ be a Feller semigroup on $C_{0}\left(\mathbb{R}_{+}\right)$with generator $\mathcal{A}$. Then for each $x_{0} \in \mathbb{R}_{+}$there exists real numbers

$$
\alpha\left(x_{0}\right) \geq 0, \quad \beta\left(x_{0}\right), \quad \gamma\left(x_{0}\right) \geq 0, \quad \delta\left(x_{0}\right) \geq 0
$$

and a nonnegative Borel measure $\nu\left(x_{0}, d y\right)$ on $\mathbb{R}_{+} \backslash\left\{x_{0}\right\}$ satisfying

$$
\int_{\mathbb{R}_{+} \backslash\left\{x_{0}\right\}} \chi^{2}\left(y-x_{0}\right) \nu\left(x_{0}, d y\right)<\infty
$$

such that for all $f \in \mathcal{D}(\mathcal{A}) \cap C_{0}^{2}\left(\mathbb{R}_{+}\right)$we have

$$
\begin{aligned}
\delta\left(x_{0}\right) \mathcal{A} f\left(x_{0}\right)= & \alpha\left(x_{0}\right) f^{\prime \prime}\left(x_{0}\right)+\beta\left(x_{0}\right) f^{\prime}\left(x_{0}\right)-\gamma\left(x_{0}\right) f\left(x_{0}\right) \\
& +\int_{\mathbb{R}_{+} \backslash\left\{x_{0}\right\}}\left(f(y)-f\left(x_{0}\right)-f^{\prime}\left(x_{0}\right) \chi\left(y-x_{0}\right)\right) \nu\left(x_{0}, d y\right) .
\end{aligned}
$$

Furthermore if $\alpha\left(x_{0}\right)=\beta\left(x_{0}\right)=\gamma\left(x_{0}\right)=\delta\left(x_{0}\right)=0$ then $\nu\left(x_{0}, \mathbb{R}_{+} \backslash\left\{x_{0}\right\}\right)>0$.

Actually, this lemma holds for any positive contraction semigroup $\left(P_{t}\right)$ on $B\left(\mathbb{R}_{+}\right)$. 
Proof. The proof follows [23, Theorem 9.5.1]. Let $p_{t}(x, d y)$ denote the transition function corresponding to $\left(P_{t}\right)$, that is

$$
P_{t} f(x)=\int_{\mathbb{R}_{+}} f(y) p_{t}(x, d y), \quad \forall f \in B\left(\mathbb{R}_{+}\right) .
$$

Fix $x_{0}$ in $\mathbb{R}_{+}$and let $f \in \mathcal{D}(\mathcal{A}) \cap C_{0}^{2}\left(\mathbb{R}_{+}\right)$. We decompose

$$
\begin{aligned}
\frac{1}{t}\left(P_{t} f\left(x_{0}\right)-f\left(x_{0}\right)\right)= & \frac{1}{t} \int_{\mathbb{R}_{+}}\left(f(y)-f\left(x_{0}\right)-f^{\prime}\left(x_{0}\right) \chi\left(y-x_{0}\right)\right) p_{t}\left(x_{0}, d y\right) \\
& +\frac{1}{t}\left(\int_{\mathbb{R}_{+}} \chi\left(y-x_{0}\right) p_{t}\left(x_{0}, d y\right)\right) f^{\prime}\left(x_{0}\right) \\
& +\frac{1}{t}\left(p_{t}\left(x_{0}, \mathbb{R}_{+}\right)-1\right) f\left(x_{0}\right) \\
= & \frac{1}{t} \int_{\mathbb{R}_{+} \backslash\left\{x_{0}\right\}} h\left(x_{0}, y\right) \chi^{2}\left(y-x_{0}\right) p_{t}\left(x_{0}, d y\right) \\
& +\beta_{t}\left(x_{0}\right) f^{\prime}\left(x_{0}\right)-\gamma_{t}\left(x_{0}\right) f\left(x_{0}\right),
\end{aligned}
$$

where

$$
\begin{aligned}
& \beta_{t}\left(x_{0}\right):=\frac{1}{t} \int_{\mathbb{R}_{+}} \chi\left(y-x_{0}\right) p_{t}\left(x_{0}, d y\right) \\
& \gamma_{t}\left(x_{0}\right):=\frac{1}{t}\left(1-p_{t}\left(x_{0}, \mathbb{R}_{+}\right)\right) \geq 0
\end{aligned}
$$

and

$$
h\left(x_{0}, y\right):=\frac{f(y)-f\left(x_{0}\right)-f^{\prime}\left(x_{0}\right) \chi\left(y-x_{0}\right)}{\chi^{2}\left(y-x_{0}\right)} .
$$

Notice that $h\left(x_{0},.\right) \in C_{b}\left(\mathbb{R}_{+}\right)$with $h\left(x_{0}, x_{0}\right)=\frac{1}{2} f^{\prime \prime}\left(x_{0}\right)$ and

$$
\lim _{y \rightarrow \infty} h\left(x_{0}, y\right)=-f\left(x_{0}\right)-f^{\prime}\left(x_{0}\right) .
$$

We introduce a new measure to rewrite the last integral in equation (46). Set

$$
l_{t}\left(x_{0}\right):=\frac{1}{t} \int_{\mathbb{R}_{+}} \chi^{2}\left(y-x_{0}\right) p_{t}\left(x_{0}, d y\right) \geq 0 .
$$

If $l_{t}\left(x_{0}\right)>0$ define

$$
\mu_{t}\left(x_{0}, d y\right):=\frac{1}{t l_{t}\left(x_{0}\right)} \chi^{2}\left(y-x_{0}\right) p_{t}\left(x_{0}, d y\right) .
$$

If $l_{t}\left(x_{0}\right)=0$ then set $\mu_{t}\left(x_{0}, d y\right):=\delta_{x_{0}+1}(d y)$, the Dirac measure in $x_{0}+1$. In both cases we have that $\mu_{t}\left(x_{0}, d y\right)$ is a probability measure on $\mathbb{R}_{+} \backslash\left\{x_{0}\right\}$ and we can write

$$
\begin{aligned}
\frac{1}{t}\left(P_{t} f\left(x_{0}\right)-f\left(x_{0}\right)\right)= & l_{t}\left(x_{0}\right) \int_{\mathbb{R}_{+} \backslash\left\{x_{0}\right\}} h\left(x_{0}, y\right) \mu_{t}\left(x_{0}, d y\right) \\
& +\beta_{t}\left(x_{0}\right) f^{\prime}\left(x_{0}\right)-\gamma_{t}\left(x_{0}\right) f\left(x_{0}\right) .
\end{aligned}
$$

Now we pass to the limit in equation (47). We introduce the nonnegative functions

$$
\theta_{n}\left(x_{0}\right):=l_{\frac{1}{n}}\left(x_{0}\right)+\left|\beta_{\frac{1}{n}}\left(x_{0}\right)\right|+\gamma_{\frac{1}{n}}\left(x_{0}\right) \geq 0, \quad n \in \mathbb{N} .
$$

We have to distinguish two cases:

Case i): $\liminf _{n \rightarrow \infty} \theta_{n}\left(x_{0}\right)=0$. There exists a subsequence $\left(\theta_{n_{k}}\left(x_{0}\right)\right)$ converging to zero. Since the limit of the left hand side of equation (47) exists it has to 
be zero and the lemma is proved with $\delta\left(x_{0}\right)=1$ and $\alpha\left(x_{0}\right)=\beta\left(x_{0}\right)=\gamma\left(x_{0}\right)=$ $\nu\left(x_{0}, \mathbb{R}_{+} \backslash\left\{x_{0}\right\}\right)=0$.

Case ii): $\liminf _{n \rightarrow \infty} \theta_{n}\left(x_{0}\right)>0$. There exists a subsequence $\left(\theta_{n_{k}}\left(x_{0}\right)\right)$ converging to $\theta\left(x_{0}\right)$ with $0<\theta\left(x_{0}\right) \leq \infty$. Divide both sides of equation $(47)$ by $\theta_{n_{k}}\left(x_{0}\right)$ and set $t_{k}:=1 / n_{k}$ to get

$$
\begin{aligned}
\frac{1}{\theta_{n_{k}}\left(x_{0}\right)}\left(\frac{P_{t_{k}} f\left(x_{0}\right)-f\left(x_{0}\right)}{t_{k}}\right)= & \frac{l_{t_{k}}\left(x_{0}\right)}{\theta_{n_{k}}\left(x_{0}\right)} \int_{\mathbb{R}_{+} \backslash\left\{x_{0}\right\}} h\left(x_{0}, y\right) \mu_{t_{k}}\left(x_{0}, d y\right) \\
& +\frac{\beta_{t_{k}}\left(x_{0}\right)}{\theta_{n_{k}}\left(x_{0}\right)} f^{\prime}\left(x_{0}\right)-\frac{\gamma_{t_{k}}\left(x_{0}\right)}{\theta_{n_{k}}\left(x_{0}\right)} f\left(x_{0}\right) .
\end{aligned}
$$

Since $\mu_{t_{k}}\left(x_{0}, d y\right)$ are probability measures on $\mathbb{R}_{+} \backslash\left\{x_{0}\right\}$ they converge weakly (after passing to a subsequence if necessary) to a probability measure $\mu\left(x_{0}, d y\right)$ on $\overline{\mathbb{R}}_{+}$. Moreover, by (48) the following limits exist

$$
\frac{1}{\theta_{n_{k}}\left(x_{0}\right)} \rightarrow \delta\left(x_{0}\right), \quad \frac{l_{t_{k}}\left(x_{0}\right)}{\theta_{n_{k}}\left(x_{0}\right)} \rightarrow l\left(x_{0}\right), \quad \frac{\beta_{t_{k}}\left(x_{0}\right)}{\theta_{n_{k}}\left(x_{0}\right)} \rightarrow \tilde{\beta}\left(x_{0}\right), \quad \frac{\gamma_{t_{k}}\left(x_{0}\right)}{\theta_{n_{k}}\left(x_{0}\right)} \rightarrow \tilde{\gamma}\left(x_{0}\right)
$$

and satisfy

$$
0 \leq \delta\left(x_{0}\right)<\infty, \quad 0 \leq l\left(x_{0}\right) \leq 1, \quad\left|\tilde{\beta}\left(x_{0}\right)\right| \leq 1, \quad 0 \leq \tilde{\gamma}\left(x_{0}\right) \leq 1,
$$

and

$$
l\left(x_{0}\right)+\left|\tilde{\beta}\left(x_{0}\right)\right|+\tilde{\gamma}\left(x_{0}\right)=1 .
$$

So the term with the integral in (49) converges to

$$
\begin{aligned}
l\left(x_{0}\right) \int_{\overline{\mathbb{R}}_{+}} h\left(x_{0}, y\right) \mu\left(x_{0}, d y\right)= & \frac{1}{2} l\left(x_{0}\right) \mu\left(x_{0},\left\{x_{0}\right\}\right) f^{\prime \prime}\left(x_{0}\right) \\
& +l\left(x_{0}\right) \mu\left(x_{0},\{\infty\}\right)\left(-f^{\prime}\left(x_{0}\right)-f\left(x_{0}\right)\right) \\
& +l\left(x_{0}\right) \int_{\mathbb{R}_{+} \backslash\left\{x_{0}\right\}} h\left(x_{0}, y\right) \mu\left(x_{0}, d y\right) .
\end{aligned}
$$

After all we get the representation (45) with

$$
\begin{aligned}
\alpha\left(x_{0}\right) & :=\frac{1}{2} l\left(x_{0}\right) \mu\left(x_{0},\left\{x_{0}\right\}\right) \geq 0 \\
\beta\left(x_{0}\right) & :=\tilde{\beta}\left(x_{0}\right)-l\left(x_{0}\right) \mu\left(x_{0},\{\infty\}\right) \\
\gamma\left(x_{0}\right) & :=\tilde{\gamma}\left(x_{0}\right)+l\left(x_{0}\right) \mu\left(x_{0},\{\infty\}\right) \geq 0
\end{aligned}
$$

and

$$
\nu\left(x_{0}, d y\right):=\frac{l\left(x_{0}\right)}{\chi^{2}\left(y-x_{0}\right)} \mu\left(x_{0}, d y\right) \quad \text { on } \mathbb{R}_{+} \backslash\left\{x_{0}\right\}
$$

Finally, we have to show that $\alpha\left(x_{0}\right)=\beta\left(x_{0}\right)=\gamma\left(x_{0}\right)=\delta\left(x_{0}\right)=0$ implies $\nu\left(x_{0}, \mathbb{R}_{+} \backslash\left\{x_{0}\right\}\right)>0$. For case i) there is nothing to prove since $\delta\left(x_{0}\right)=1$ anyway. For case ii) it follows immediately from (50) that $l\left(x_{0}\right)>0$, which by definition of $\gamma\left(x_{0}\right)$ implies that $\mu\left(x_{0},\{\infty\}\right)=0$. Furthermore $\mu\left(x_{0},\left\{x_{0}\right\}\right)=0$ by definition of $\alpha\left(x_{0}\right)$. This means $\mu\left(x_{0}, \mathbb{R}_{+} \backslash\left\{x_{0}\right\}\right)=1$ and hence $\nu\left(x_{0}, \mathbb{R}_{+} \backslash\left\{x_{0}\right\}\right)>0$.

Acknowledgement. I am grateful to J. Zabczyk for initiating the problem in discrete time. I also thank D. Duffie and W. Schachermayer for their interest and comments. Financial support from Credit Suisse is gratefully acknowledged. 


\section{REFERENCES}

[1] N. H. Bingham, C. M. Goldie, and J. L. Teugels, Regular variation, Cambridge University Press, Cambridge, 1987.

[2] T. Björk, Y. Kabanov, and W. Runggaldier, Bond market structure in the presence of marked point processes, Math. Finance 7 (1997), no. 2, 211-239.

[3] R. G. Brown and S. M. Schaefer, Interest rate volatility and the shape of the term structure, Phil. Trans. R. Soc. Lond. A 347 (1994), 563-576.

[4] R. Courant and D. Hilbert, Methoden der mathematischen Physik, Springer-Verlag, Berlin, 1993, Vierte Auflage.

[5] J. Cox, J. Ingersoll, and S. Ross, A theory of the term structure of interest rates, Econometrica 53 (1985), 385-408.

[6] D. Duffie and N. Gârleanu, Risk and valuation of collateralized debt obligations, forthcoming, Financial Analysts Journal.

[7] D. Duffie and R. Kan, A yield-factor model of interest rates, Math. Finance 6 (1996), 379-406.

[8] D. Duffie, J. Pan, and K. Singleton, Transform analysis and asset pricing for affine jumpdiffusions, Working paper, Stanford University, 1999.

[9] E. B. Dynkin, Markov processes, vol. I, Springer-Verlag, Berlin, 1965.

[10] S. N. Ethier and T. G. Kurtz, Markov processes, John Wiley \& Sons Inc., New York, 1986, Characterization and convergence.

[11] W. Feller, An introduction to probability theory and its applications, second ed., vol. II, John Wiley \& Sons, 1971.

[12] D. Filipović, A note on the Nelson-Siegel family, Math. Finance 9 (1999), no. 4, 349-359.

[13] D. Filipović and J. Zabczyk, Markovian term structure models in discrete time, Preprint 601, Polish Academy of Sciences, December 1999.

[14] N. Ikeda and S. Watanabe, Stochastic differential equations and diffusion processes, NothHolland, Amsterdam, 1981.

[15] N. L. Johnson and S. Kotz, Distributions in statistics. Continuous univariate distributions. 2., Houghton Mifflin Co., Boston, Mass., 1970.

[16] K. Kawazu and S. Watanabe, Branching processes with immigration and related limit theorems, Theory Probab. Appl. 16 (1971), 36-54.

[17] M. Musiela and M. Rutkowski, Martingale methods in financial modelling, Applications of Mathematics, vol. 36, Springer-Verlag, Berlin-Heidelberg, 1997.

[18] D. Revuz and M. Yor, Continuous martingales and Brownian motion, Grundlehren der mathematischen Wissenschaften, vol. 293, Springer-Verlag, Berlin-Heidelberg-New York, 1994.

[19] L. C. G. Rogers and D. Williams, Diffusions, Markov processes, and martingales, second ed., vol. 1, John Wiley \& Sons, 1994.

[20] L. C. G. Rogers and O. Zane, Saddlepoint approximations to option prices, Ann. Appl. Probab. 9 (1999), no. 2, 493-503.

[21] T. Shiga and S. Watanabe, Bessel diffusions as a one-parameter family of diffusion processes, Z. Wahrscheinlichkeitstheorie verw. Geb. 27 (1973), 37-46.

[22] M. L. Silverstein, A new approach to local times, J. Math. Mech. 17 (1967/1968), 1023-1054.

[23] K. Taira, Diffusion processes and partial differential equations, Academic Press, London, 1988.

[24] A. D. Venttsel', On boundary conditions for multidimensional diffusion processes, Theory Probab. Appl. 4 (1959), 164-177.

[25] S. Watanabe, On two dimensional Markov processes with branching property, Trans. Amer. Math. Soc. 136 (1969), 447-466.

Departement Mathematik, ETH, RÄmistrasse 101, CH-8092 Zürich, Switzerland

E-mail address: filipo@math.ethz.ch 